\title{
Laser treatment of traumatic scars: a military perspective
}

\author{
Peter R Shumaker, MD
}

\section{Abstract}

Advancements in medical treatment and transport over more than a decade of conflict have resulted in unprecedented survival rates for service members despite catastrophic injuries. Enhanced survival has created an unprecedented need for comprehensive rehabilitation and transition services. Though far from the exclusive domain of military dermatologists, military medicine has had a prominent role in integrating cutaneous procedural techniques into the rehabilitation of traumatically injured patients for a variety of reasons. The introduction of fractional laser technology in the mid-2000's has stimulated a revolution in scar treatment, and is gradually remodeling the fields of procedural dermatology and trauma rehabilitation both inside and outside of the military. This manuscript will provide a brief review of common cutaneous procedures applicable to rehabilitation, with an emphasis on ablative fractional laser resurfacing of scars and contractures.

\section{Semin Cutan Med Surg 34:17-23 @ 2015 Frontline Medical} Communications

A fter more than a decade of conflict, increased survival on the battlefield has pushed thousands of severely injured service members into the military medical system. The influx of many young, motivated, and otherwise healthy patients has raised not only the overall need, but the ultimate goals for functional recovery to approximate preinjury levels. The emergence of military centers of excellence in trauma rehabilitation has facilitated multidisciplinary collaboration to help coordinate this effort. ${ }^{1}$ Technologies designed primarily for cosmetic applications, such as fractional laser resurfacing and laser hair reduction, have been adapted for consistent functional enhancement. The collaborative environment and a relative sheltering from reimbursement issues have facilitated the advancement and early adoption of these techniques at various military centers. As a result of these factors, dermatologists are emerging as routine partners in posttrauma care to the great benefit of the specialty and to trauma patients.

Trauma and scarring are (at present) inextricably linked; disfigurement, diminished function, and symptoms such as pain and itch decrease the quality of life for millions of patients worldwide. Among the most exciting and potentially far-reaching recent

Chairman, Dermatology, Naval Medical Center San Diego, California. Disclosures: Dr Shumaker has completed and submitted the ICMJE Form for Disclosure of Potential Conflicts of Interest and none were reported. Correspondence: Peter R. Shumaker, MD; Dermatology Department; Naval Medical Center; 34800 Bob Wilson Dr., Suite 300; San Diego, CA 92134. E-mail: peter.shumaker@med.navy.mil breakthroughs in scar management is the advent of ablative fractional laser resurfacing (AFR). Other well-established modalities such as vascular-specific lasers and corticosteroids continue to be integral to treatment. The emergence of the improvised explosive device as a pervasive weapon in Iraq and Afghanistan and its association with extremity amputations has highlighted the potential role for other cutaneous procedural modalities to reduce hair and sweat to facilitate prosthetic use, and to treat traumatic tattoos. It should be recognized that most of the patients who will benefit from these advances will never wear a uniform. Interest and expertise in these procedures is by no means limited to the military, and burn centers and other institutions have been a major driving force in these breakthroughs. This trend will only accelerate as these techniques continue to gain wider acceptance around the globe. This manuscript provides a review of common cutaneous procedures in trauma rehabilitation, with an emphasis on AFR for traumatic scars and scar contractures at a single military center. While evidence of the benefit of these techniques is accumulating in the literature, it should be acknowledged that many recommendations herein are still based largely on expert opinion.

\section{Laser treatment of scars}

Simply stated, laser scar revision is based on the induction of a controlled thermal injury to initiate a favorable healing and remodeling response in the area of interest. The type and extent of the injury determines the laser wavelength, delivery method, and operatordetermined settings such as fluence, treatment depth, and density. Excellent reviews of laser-tissue interactions in this context have been published previously and will not be recapitulated here., ${ }^{2,3}$ The most common modalities used for scar treatment include laser and light devices that target hemoglobin in erythematous scars, and lasers that target water in the case of fractional and full-field ablative and nonablative fractional lasers. Vascular-specific and full-field ablative lasers have been used for decades in scar treatment. ${ }^{4,5}$ However, their application for extensive traumatic scars, especially function-limiting scar contractures, has been hindered by modest efficacy or excessive thermal injury when implemented aggressively. For reasons still not completely understood, fractionation of tissue vaporization and coagulation across the treatment field has revolutionized scar management by increasing attainable treatment depths while minimizing contiguous thermal injury. Vascular laser and light devices in addition to ablative fractional lasers form the core duo of devices for the treatment of significant traumatic scars in the author's current practice.

\section{Vascular lasers}

Vascular-specific laser and light devices, particularly the $585 \mathrm{~nm}$ and $595 \mathrm{~nm}$ pulsed-dye laser (PDL), have been used successfully for decades in the treatment of traumatic and surgical scars. ${ }^{4,6,7}$ 
While the PDL has accumulated the greatest body of supporting literature, other devices that target hemoglobin absorption peaks such as the $532 \mathrm{~nm}$ potassium titanyl phosphate laser and intensepulsed light devices can also be effective. In contrast to other applications, lower treatment fluences are generally employed in scar treatment as a reflection of its likely reliance on tissue remodeling rather than tissue destruction. ${ }^{2}$ For example, when using a common $595 \mathrm{~nm}$ PDL (Vbeam, Syneron \& Candela, Wayland, Massachusetts, USA), the author generally uses fluences in the range of 7 to $8 \mathrm{~J} / \mathrm{cm}^{2}$ with a spot size of $7 \mathrm{~mm}$ and a pulse duration of $1.5 \mathrm{~ms}$ to generate an endpoint of minimal purpura. Erythema can be a helpful surrogate for overall scar activity and associated symptoms such as pain and itch, and for potentially incipient pathological scarring. As such, in the author's practice, vascular devices are integrated into scar treatment using a simple one-step algorhythm: Is it red? As in years past, the benefits of integrating vascular-specific devices include their consistent efficacy in reducing symptoms parallel with erythema, tolerability often without anesthesia, and the ability to treat large areas in a single session.

\section{Nonablative fractional resurfacing}

Scar treatment was serendipitously altered forever when the concept of fractional photothermolysis was introduced into the literature in $2004 .{ }^{8}$ Moderate water absorption in the midinfrared wavelengths induces tissue coagulation in a pixelated pattern, ostensibly allowing increased penetration depth (up to approximately $1.5 \mathrm{~mm}$ ) while minimizing cumulative injury throughout the treatment field. Nonablative fractional resurfacing (NAFR) has also shown promise in the treatment of traumatic scars in a number of prospective studies, particularly in the improvement in scar appearance and texture. ${ }^{9-11}$ While head-to head studies comparing ablative to nonablative resurfacing on the treatment of traumatic scars and contractures are generally lacking, a recent consensus report advocated AFR over NAFR for the treatment of thicker scars with associated functional deficits. ${ }^{2}$ When applied to traumatic scars, NAFR is likely best employed for atrophic scars, or when treatment goals consist mainly of improving dyspigmentation and textural irregularities. As in most of the modalities described herein, multiple treatments for cumulative improvements are expected.

\section{Ablative fractional resurfacing}

AFR is potentially transformative in the field of trauma rehabilitation, offering a paradigm-shifting minimally invasive yet consistently effective treatment for symptomatic hypertrophic scars and contractures. A review of AFR for traumatic scar treatment was published out of our institution in 2012. ${ }^{3}$ At that time, supporting evidence for efficacy of the procedure for functional and cosmetic improvements in scars and scar contractures was positive but overwhelmingly anecdotal. While still limited, there are an accumulating number of reports in the literature including increasingly rigorous evaluations to include retrospective and prospective studies. ${ }^{12-16}$ Ozog et al recently published the first description of histopathologic improvements in tissue architecture and collagen composition that offer early clues to the efficacy of the procedure. ${ }^{13}$ Recent reviews and a consensus report also point to increasing integration of the technique into treatment paradigms, with plenty of room for larger and higher-quality studies to refine our knowledge of the technique., ${ }^{2,17-19}$ Recognition of the potential functional ben- efits of the technique has been increasing, and reports have been published in top journals representing at least eight different medical specialties. ${ }^{1,12,14,15,20-23}$

Fractional lasers are the first to offer tunable depths of penetration and treatment density, an important level of control in the highly variable environment after trauma. But, what should we do with this newfound control? At present, the literature offers little expert opinion concerning optimal settings and techniques in various clinical situations. Current ablative fractional devices provide depths of penetration of up to approximately $4 \mathrm{~mm}$, while nonablative devices coagulate tissue to a depth up to approximately $1.5 \mathrm{~mm}$. This difference may explain a portion of the perceived advantage in efficacy for AFR in the thickest scar contractures. While the optimal treatment depth for individual scar types has not been elucidated, a consensus report by Anderson et al advocated a treatment depth proportional to and without exceeding scar thickness. ${ }^{2}$

In addition to treatment depth, the laser operator may also select the density of ablative columns. Safe treatment mandates a minimization of excessive thermal injury, an increase in treatment depth, and like a see-saw, mandates a corresponding reduction in treatment density. When treating thick scars and contractures, the author generally uses higher fluences for greater penetration depth and a low corresponding treatment density which is frequently the lowest setting. In the opinion of the author, little is gained by increasing treatment density beyond low levels. Since density settings vary from platform to platform, the meaning of "low" will also vary. However, for most devices, density should remain below $10 \%-15 \%$ for traumatic scar treatment, particularly for contractures.

\section{Optimal laser characteristics}

Early indications are that ablative fractional resurfacing with ablative column diameters in the range of hundreds of microns (ie, microfractional injuries) will result in healing without additional fibrosis in both scars and normal skin. This threshold seems to be in the vicinity of $500 \mu \mathrm{m}$ or less. ${ }^{2,13,24}$ While the optimal microcolumn diameter is unknown, it is logical that it should be no more than a few hundred microns. Pulse width is also an important consideration as pulse duration significantly longer than the thermal relaxation time of tissue, approximately $1 \mathrm{~ms}$, could result in excessive thermal damage and potentially worsening of scarring. . $^{2,3}$

Ablative fractional lasers come in two main types, the 10600 $\mathrm{nm}$ carbon dioxide $\left(\mathrm{CO}_{2}\right)$ and the $2940 \mathrm{~nm}$ erbium: yttrium-aluminum-garnet (Er:YAG). Due to its higher avidity for absorption by tissue water, Er:YAG ablative columns are associated with a narrower rim of thermal coagulation than $\mathrm{CO}_{2}$. As a result, Er:YAG laser treatments have a greater propensity for bleeding than $\mathrm{CO}_{2}$ treatments. Comparative studies for traumatic scar treatment have not yet been performed, and the optimal level of coagulation surrounding the ablative column has not been defined. Indeed, the efficacy of skin needling (percutaneous collagen induction) in scar remodeling indicates that thermal injury is only part of the story. ${ }^{25}$ However, if we extrapolate from comparative full-field resurfacing and incisional studies, an appropriate degree of coagulation likely has a stimulative effect for the overall remodeling response., ${ }^{3,26}$

Future studies will be required to match the optimal platform and settings to specific indications. However, characteristics such as a narrow microcolumn diameter, short-pulse width, and ample 
penetration depth appear to be good starting points to consider in an ablative fractional platform dedicated to scar management. Treatments should be applied conservatively with low treatment densities, particularly when high-pulse energies are selected for enhanced treatment depth in thicker scars.

\section{Ablative fractional laser technique}

Traumatic injuries are complex, as are the resulting scars. Therefore, selected treatment parameters and combinations will vary in each specific location in each treatment session. It is premature to definitively link the optimal laser, settings, timing, and scar type, as further research is required to elaborate these details more completely. What follows are general recommendations based on the experience at a single institution successfully integrating AFR to mitigate scars of a wide variety of ages and origins. The overall technique is similar to that described previously. ${ }^{3}$ The discussion below focuses primarily on large traumatic scars in the context of significant trauma (ie, those previously not considered within the domain of dermatologists). Smaller surgical and traumatic scars may be amenable to more aggressive treatment and abbreviated time courses for intervention, perhaps even on the day of surgery. ${ }^{27,28}$ It should also be understood that in the author's institution, laser scar management following major trauma is generally performed as a component of a comprehensive rehabilitative effort including multiple elements such as case management, mental health support, physical, occupational, and recreational therapy, prosthetic support, gait training, and surgical and nonsurgical medical evaluation. In addition, traditional methods in management such as physical therapy and options for surgical revision remain central to quality care.

\section{Pain management}

In the practice of the author, the majority of AFR treatments are performed in the outpatient setting without sedation. For patients who have often endured numerous reconstructive surgeries prior to presentation, the option for an effective procedure outside of the operating room is highly popular. Frequently treated areas (especially split-thickness skin graft sites) may be anesthetic or hypoesthetic in the first months and years after trauma. Furthermore, in the author's experience, postprocedure discomfort is minimal and many patients are willing to endure a few minutes of (mitigated) discomfort to spare a day of inconvenience including mental cloudiness and nothing-by-mouth. There are, however, undoubtedly patients who are good candidates for general anesthesia or conscious sedation based on their age, the extent of their injuries, degree of sensitivity, or background factors such as posttraumatic stress. Regardless, the treating physician should be aware that the feeling of heat, burning smell, or the loud sounds associated with the procedure may serve as triggers for anxiety and should be anticipated and mitigated accordingly. Pain in the days following the procedure is usually minimal and supplemental oral medications are not generally required. In fact, in a recent retrospective study by Perry et al involving AFR for functional improvements at the wrist and forearm, a majority of patients reported improved pain in the days, weeks, and months following AFR. ${ }^{12}$

In the author's practice, anesthesia is most frequently provided with an off-the-shelf topical anesthetic such as $4 \%$ lidocaine cream under occlusion for an hour or more before treatment, either alone or in combination with other methods. While potent compounded combination anesthetics can be more effective, the potential for systemic toxicity must be factored in when applied over large areas with variable absorption. This is frequently supplemented at the time of treatment with the application of a cold pack prior to a series of pulses in a given area. For appropriate locations, infiltration with local anesthetic, focal nerve blocks, or regional blocks can also be considered. Of note, anatomic disruption after trauma can lead to a variable response to nerve blocks, and tumescent anesthesia must be approached with extreme caution in areas with restrictive scarring and potential damage to regional lymphatics.

\section{Time to initiate treatment}

In the view of the author, scars of virtually any age should be considered candidates for AFR, from about 3 months to 3 decades or more after injury. For a variety of factors including transport and the transition from inpatient to outpatient management of their injuries, patients have been evaluated in Dermatology beginning as early as approximately 2 to 3 months after injury or final reconstructive surgery. This timeframe appears to be favorable for initiating intervention, as incipient contractures and chronic wounds are declaring themselves and patients are often in a state of significant transition in rehabilitation, such as initiating prosthetic use. Younger scars (less than a year or so after injury) are more susceptible to breakdown than more mature scars and should be treated judiciously in both laser settings and combination treatments.

The potential opportunity for procedural intervention for early scar contractures deserves special comment. Early, active procedural contracture mitigation would represent a major worldwide paradigm shift in scar management with positive implications for the system of trauma rehabilitation. Traditional paradigms for surgical scar revision typically dictate waiting a year or more after injury to allow for spontaneous scar maturation. ${ }^{29,30}$ In the view of the author, the favorable combination of safety and efficacy of the procedure offers a sturdy basis for future study, and a potential quantum leap above existing conservative measures. Documenting efficacy through controlled trials will be necessary to definitively distinguish laser-mediated changes from spontaneous improvement in the early recovery period. However, the consistent improvements within days of each procedure and the almost universal positive responses from patients offer reasons to be optimistic. Avoidance or a reduction in the severity of permanent disability and symptoms from contractures for even a fraction of patients would be potentially transformative.

\section{Timing between treatments}

AFR for traumatic scars should be considered a course rather than a single treatment. In the practice of the author, repeated treatments after a minimum interval of 6 to 8 weeks have resulted in incremental improvements and are well tolerated. While not yet supported with studies, the majority of functional improvements seems to be realized within the first 3 to 5 treatments, and is optimized when combined with concurrent physical and occupational therapy and other adjunctive treatments such as vascular lasers and corticosteroids.

\section{Treatment parameters}

Favorable laser characteristics (narrow microcolumn width and 

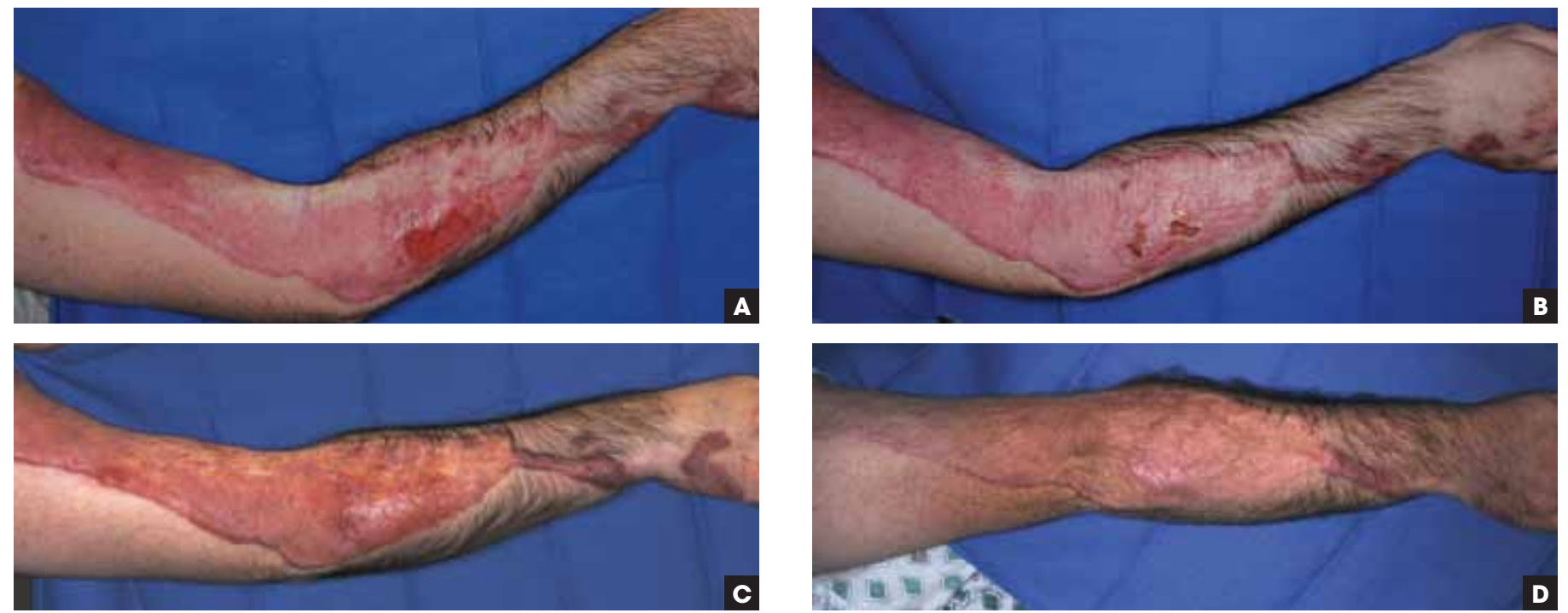

FIGURE 1. A) A man in his 20s with a moderate flexion contracture approximately 3 months after a motorcycle accident requiring split-thickness skin grafts during reconstruction. A large nonhealing erosion adjacent to an area of tension is also observed. B) Approximately 1 month after his initial ablative fractional laser treatment, the patient demonstrates interval improvements in range of motion and wound healing. C) Approximately 2 months after his initial ablative fractional laser treatment, cumulative improvements in range of motion and wound healing are observed. D) The patient manifests normal range of motion and complete re-epithelialization approximately one year after initiating a course of three ablative fractional laser treatments and laser-assisted corticosteroid delivery. The patient also participated in regular occupational therapy.

pulse width) and treatment parameters (trends toward lower density and treatment depth proportional to scar thickness) have been described above. A variety of fractional ablative devices are available, and specific treatment variables will depend on the nuances of the particular device. While ultrasound can be useful for ascertaining scar thickness; in the experience of the author, simple palpation is sufficient to estimate scar thickness and determine laser settings outside of research settings. Current ablative devices can achieve depths of penetration up to approximately $4 \mathrm{~mm}$. However, in most clinical settings, the author employs pulse energies corresponding to a treatment depth of $1 \mathrm{~mm}$ to $2 \mathrm{~mm}$ and the lowest or one of the lowest density settings. For lasers delivering a "stamped" pattern of microcolumns, treatments are generally delivered in a single pass without overlap to minimize thermal injury. Beware of excessive treatment depth in devices with a relatively large microcolumn width, as the potential for scarring likely increases with the size of the generated wound.

\section{Periprocedural care}

Within minutes, hive-like swellings and erythema generally develop in the area of treatment. There is often a scant serous discharge, and a variable but generally small amount of bleeding depending on the treatment depth, laser type, and the age of the scar. Bleeding is generally increased in younger scars and when an Er:YAG laser is used. Regardless, it usually abates within several minutes after treatment. Treatments in mature scars with a $\mathrm{CO}_{2}$ laser platform generally result in minimal bleeding. Prophylactic oral antibiotics are used infrequently by the author since the posttreatment infection rate appears to be extremely low. However, antivirals should be considered when treating around the face. After treatment, a thin layer of petrolatum is applied and application is continued for approximately 2 days or until the epidermal barrier is recon- stituted. Nonstick dressings are applied for convenience. Patients are allowed to shower within the first day, though submersion is not recommended until the epidermal barrier is restored. There are generally no limitations in activity, and the resumption of physical therapy is highly encouraged within the first day or two after treatment to help maximize the results of subsequent scar remodeling.

\section{Treatment combinations and adjuncts}

Traumatic scars are frequently complex, and a single patient or even a single area may have foci of scar hypertrophy, atrophy, erosions, contracture, erythema, dyspigmention, and textural irregularity. While AFR can address all of these issues to a degree, it is common and desirable to employ multiple modalities such as surgical revision, tissue fillers, vascular lasers, nonablative fractional lasers, and corticosteroids into treatment concurrently or in series. Furthermore, related issues such as folliculitis and abnormal hair distribution, traumatic tattoos, and excessive sweating (such as under prosthetic liners) can be treated with adjunctive procedures such as laser hair reduction, short-pulsed (Q-switched) lasers, and botulinum toxin, respectively.

\section{Combining AFR and surgical revision}

The question is not whether one should always choose AFR or surgical revision for scar contractures - in the view of the author they are often both essential and complementary in providing optimal care. While nonsurgical treatments such as AFR can mitigate mild or even moderate scar contractures (Figures 1A-1D), severe contractures with devastating functional consequences (such as symptomatic ectropion) or obvious tissue deficit may require timely surgical intervention such as flaps, grafts, and tissue substitutes. Even in situations ultimately requiring surgical intervention, AFR can be a helpful adjunct with a series of treatments prior to surgery, 

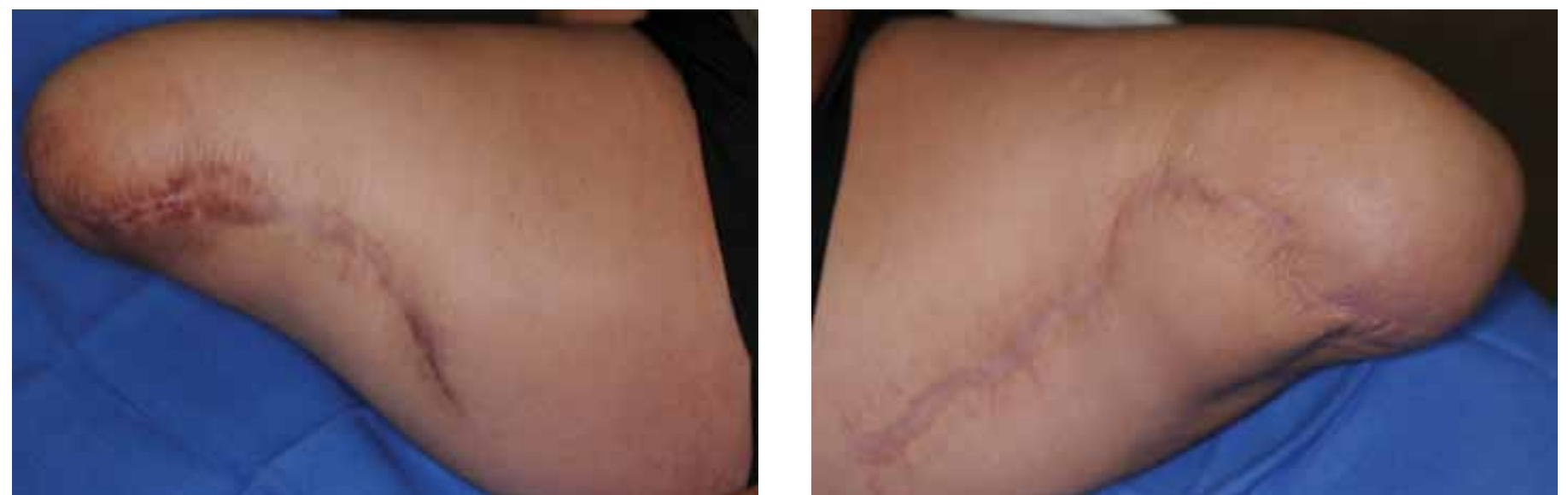

FIGURE 2. A man in his 20s with bilateral above-knee amputations after a course of treatment including multiple sessions of ablative fractional laser resurfacing to related scars and laser hair reduction in the area encompassed by the prosthetic liner. These residual limbs propelled their owner to the summit of Mount Kilimanjaro approximately 18 months after injury from an improvised explosive device.

at the time of surgery, and to help optimize results after surgery. In situations not requiring urgent surgical correction or within the first year after injury, there seems to be little to lose by initiating a course of AFR. In some cases, AFR and other adjuncts can ultimately obviate the need for surgery, and pretreatment can also enhance the quality of the tissue in the anticipated surgical field. Large traumatic scars (such as after burns) may have focal areas of contracture amenable to surgery, while the remainder can benefit from AFR for a synergistic response.

\section{Laser-assisted delivery}

One of the most exciting developments related to the advent of ablative fractional laser technology is laser-assisted delivery of various substances uniformly to the desired site of action below the epidermal barrier. ${ }^{31}$ Pertaining to scar treatment, topically applied corticosteroids and synthetic tissue fillers have recently been reported to be successful adjuncts in the treatment of hypertrophic and atrophic scars, respectively. ${ }^{32,33}$ In the experience of the author, pretreatment of a tethered or restrictive scar with AFR prior to and concurrent with augmentation with fillers, such autologous fat can be synergistic in part by increasing the pliability of the overlying scar and perhaps by modulating the subsequent remodeling response. Current and future research combining AFR with various autologous cells such as fibroblasts, adipose and adipose-derived stem cells, and bone-marrow derived stem cells may lead to additional breakthroughs in wound healing and tissue regeneration. ${ }^{34-36}$

\section{Reduction of hair and sweat}

Similar to the role of AFR for scar management, it should be recognized that not every laser hair reduction procedure and every botulinum toxin injection is strictly "cosmetic." Injuries due to improvised explosive devices and other sources of trauma during more than a decade of conflict have resulted in thousands of young, otherwise healthy patients with single and multiple amputations. ${ }^{1}$ Developing procedures to enhance the quality and the durability of the skin in the residual limbs and ultimately increase the duration and intensity of activities in prosthetics is very much a functional issue. In the experience of the author, laser hair reduction can con- sistently enhance the quality of life of amputees by improving the fit and comfort of prosthetic devices and reducing the incidence of folliculitis and abscess formation associated with prosthetic liners (Figure 2). Furthermore, hair bundling due to local scarring and/ or alterations in the quality or location of terminal hair associated with reconstructive procedures may lead to cosmetic or functional issues that can be mitigated with hair reduction.

Excessive sweating can be a significant problem for amputees, increasing the possibility of skin maceration under the liner and decreasing time in the prosthetic. In extreme cases, the prosthetic may literally fall off during ambulation. Interestingly, in the experience of the author, many patients report at least a temporary reduction in excessive sweating in the residual treated limb after a course of laser hair treatment. A small pilot study out of our institution demonstrated a statistically significant reduction in axillary sweating after laser hair reduction in patients with focal axillary hyperhidrosis. ${ }^{37}$ Botulinum toxin A injection is a US Food and Drug Administration approved treatment for focal axillary hyperhidrosis, and has also been reported as an effective (though temporary) off-label treatment for amputees with excessive sweating in the residual limb. ${ }^{38}$ Procedural treatments using current and developing technologies (such as microwave and laser devices) for the reduction of hyperhidrosis offer some hope for longer-lasting sweat reduction in the future. Larger controlled studies will be required to confirm these observations and better elucidate how these procedures can be integrated into the management of trauma patients in the future.

\section{Case example}

A man in his 20's suffered a friction injury to the right lateral arm associated with a motorcycle crash requiring split-thickness skin grafting during reconstruction. He presented to Dermatology with complaints of persistent decreased range of motion and nonhealing wounds approximately 3 months after injury despite dedicated wound care and occupational therapy several times a week. Examination revealed a moderate flexion contracture and large erosion on the dorsal surface of the distal forearm (Figure 1A). While moderate-to-severe flexion contractures often require surgical intervention for an optimal response, the relatively short interval 
after injury and our previous positive experience with AFR in the early period after injury led us to initiate a course in this case. After informed consent, topical $4 \%$ lidocaine cream was applied to the treatment area for 1 hour under occlusion. During the procedure, anesthesia was supplemented with a cold pack applied prior to a series of pulses. The entire grafted area was then treated with an ablative fractionated $10600 \mathrm{~nm} \mathrm{CO}_{2}$ laser (Lumenis Ultrapulse, Deep FX, Yokneam, Israel) in a single pass without overlap at pulse energy settings of $40 \mathrm{~mJ}$ to $50 \mathrm{~mJ}$, depending on estimated scar thickness, and a density of 5\%. Triamcinolone acetonide suspension at a concentration of $40 \mathrm{mg}$ per $\mathrm{ml}$ was then applied to hypertrophic areas within minutes of laser treatment. Petrolatum ointment and nonstick bandages were then applied. The patient continued to apply petrolatum for two days following the procedure and resumed occupational therapy the day after the procedure. The patient returned for follow-up 1 month and again at 2 months after his initial treatment, demonstrating interval improvements in range of motion, wound healing, pliability, and texture (Figures 1B and 1C). The patient received 2 additional treatments at approximately 6-8 week intervals using a similar technique. Due to moderate discomfort and associated procedural anxiety, subsequent treatments were performed under general anesthesia. Pulse energies varied from 40 $\mathrm{mJ}$ to $80 \mathrm{~mJ}$ and densities from 5\%-3\%, respectively, with increasing estimated scar thickness. At follow-up approximately 1 year after his initial treatment, the patient exhibited essentially normal extension and significant interval improvements in color, texture, and pliability (Figure 1D). Ultimately, surgical intervention was not required to restore full range of motion in this case.

\section{Conclusions}

An infusion of young, motivated, and otherwise healthy trauma patients into the military medical system has increased the expectations for rehabilitation to include preinjury levels of function in many cases. Recent advancements in technology and applications, influenced heavily by dermatologists both inside and outside of the military, have introduced minimally invasive procedures with significant functional impact to assist in this effort. While recent reports are replete with descriptions of subjective and objective functional improvement associated with AFR, existing scar scales such as the Patient and Observer Scar Assessment Scale and Vancouver Scar Scale are insufficient to document these changes because they do not account significantly for improvements in function. ${ }^{39}$ Future research must include larger controlled studies to confirm the safety and efficacy of these procedures and elaborate on optimal treatment parameters and combinations. Furthermore, the comparison of various interventions must be accompanied by the introduction of a more comprehensive method to measure and compare functional outcomes. The importance of cosmetic enhancement is certainly not diminished by the emergence of function as a new endpoint for therapy, and the restoration of appearance will continue to be a priority of multidisciplinary treatment teams.

Disclaimer: The views expressed in this article are those of the author and do not necessarily reflect the official policy or position of the Department of the Navy, Department of Defense, or the United States Government. Dr Shumaker is a military service member. This work was prepared as part of his official duties. Title 17, USC,
$\S 105$ provides that 'Copyright protection under this title is not available for any work of the United States Government.' Title 17, USC, $\S 101$ defines a US Government work as a work prepared by a military service member or employee of the US Government as part of that person's official duties.

\section{References}

1. Sheridan RL, Shumaker PR, King DR, Wright CD, Itani KM, Cancio LC. Case records of the Massachusetts General Hospital. Case 15-2014. A man in the military who was injured by an improvised explosive device in Afghanistan. $N$ Engl J Med. 2014;370(20):1931-1940.

2. Anderson RR, Donelan MB, Hivnor C, et al. Laser treatment of traumatic scars with an emphasis on ablative fractional laser resurfacing: consensus report. JAMA Dermatol. 2014;150(2):187-193.

3. Uebelhoer NS, Ross EV, Shumaker PR. Ablative fractional resurfacing for the treatment of traumatic scars. Semin Cutan Med Surg. 2012;31(2):110-120.

4. Alster TS. Improvement of erythematous and hypertrophic scars by the $585-\mathrm{nm}$ flashlamp-pumped pulsed dye laser. Ann Plast Surg. 1994;32(2):186-190.

5. Garrett AB, Dufresne RG Jr, Ratz JL, Berlin AJ. Carbon dioxide laser treatment of pitted acne scarring. J Dermatol Surg Oncol. 1990;16(8):737-740.

6. Parrett BM, Donelan MB. Pulsed dye laser in burn scars: current concepts and future directions. Burns. 2010;36(4):443-449.

7. Vrijman C, van Drooge AM, Limpens J, et al. Laser and intense pulsed light therapy for the treatment of hypertrophic scars: a systematic review. Br J Dermatol. 2011;165(5):934-942.

8. Manstein DD, Herron GS, Sink RK, Tanner H, Anderson RR. Fractional photothermolysis: a new concept for cutaneous remodeling using microscopic patterns of thermal injury. Lasers Surg Med. 2004;34(5):426-438.

9. Taudorf EH, Danielson PL, Paulsen IF, et al. Non-ablative fractional laser provides long-term improvement of mature burn scars - A randomized controlled trial with histological assessment [published online ahead of print August 22, 2014]. Lasers Surg Med. doi: 10.1002/lsm.22289.

10. Haedersdal M, Moreau KE, Beyer DM, Nymann P, Alsbjørn B. Fractional nonablative $1540 \mathrm{~nm}$ laser resurfacing for thermal burn scars: a randomized controlled trial. Lasers Surg Med. 2009;41(3):189-195.

11. Waibel J, Wulkan AJ, Lupo M, Beer K, Anderson RR. Treatment of burn scars with the 1,550 $\mathrm{nm}$ nonablative fractional Erbium Laser. Lasers Surg Med. 2012;44(6):441-446.

12. Perry A, Elston J, Reynolds H, et al. Ablative fractional photothermolysis in the treatment of scar contractures of the wrists and forearms: a retrospective data analysis. J Am Acad Dermatol. 2014;71(4):841-842.

13. Ozog DM, Liu A, Chaffins ML, et al. Evaluation of clinical results, histological architecture, and collagen expression following treatment of mature burn scars with a fractional carbon dioxide laser. JAMA Dermatol. 2013;149(1):50-57.

14. Hultman CS, Friedstat JS, Edkins RE, Cairns BA, Meyer AA. Laser resurfacing and remodeling of hypertrophic burn scars: the results of a large, prospective, beforeafter cohort study, with long-term follow-up. Ann Surg. 2014;260(3):519-529; discussion 529-532.

15. Hultman CS, Edkins RE, Wu C, Calvert CT, Cairns BA. Prospective, before-after cohort study to assess the efficacy of laser therapy on hypertrophic burn scars. Ann Plast Surg. 2013;70(5):521-526.

16. Khandelwal A, Yelvington M, Tang X, Brown S. Ablative fractional photothermolysis for the treatment of hypertrophic burn scars in adult and pediatric patients: a single surgeon's experience. J Burn Care Res. 2014;35(5):453-463.

17. Gold MH, Berman B, Clementoni MT, Gauglitz GG, Nahai F, Murcia C. Updated international clinical recommendations on scar management: part 1 -evaluating the evidence. Dermatol Surg. 2014;40(8):817-824.

18. Gold MH, McGuire M, Mustoe TA, et al; International Advisory Panel on Scar Management. Updated international clinical recommendations on scar management: part 2 - algorithms for scar prevention and treatment. Dermatol Surg. 2014;40(8):825-831.

19. Friedstat JS, Hultman CS. Hypertrophic burn scar management: what does the evidence show? A systematic review of randomized controlled trials. Ann Plast Surg. 2014;72(6):S198-S201.

20. Kwan JM, Wyatt M, Pyo J, Uebelhoer NS, Shumaker PR. Functional improvement following ablative fractional laser treatment of a scar contracture. PMR. 2011;3(10):986-987.

21. Kroonen L, Shumaker PR, Kwan JM, Uebelhoer NS, Hofmeister E. Treatment of split-thickness skin grafted forearm scar contractures with a carbon dioxide laser protocol: 3 case reports. J Hand Surg Am. 2013:38(11):2164-2168.

22. Krakowski AC, Goldenberg A, Eichenfield LF, Murray JP, Shumaker PR. Ablative fractional laser resurfacing helps treat restrictive pediatric scar contractures [published online ahead of print November 3, 2014]. Pediatrics. 2014;134(6):e1700e1705. doi: 10.1542/peds.2014-1586. 


\section{Shumaker}

23. Cox JA, Dainer M, Shumaker PR. Ablative fractional laser resurfacing for abdominal scar contractures in pregnancy. Obstet Gynecol. In press.

24. Hantash BM, Bedi VP, Kapadia B, et al. In vivo histological evaluation of a novel ablative fractional resurfacing device. Lasers Surg Med. 2007;39(2):96-107.

25. Aust MC, Knobloch K, Reimers K, et al. Percutaneous collagen induction therapy: an alternative treatment for burn scars. Burns. 2010;36(6):836-843.

26. Ross EV, Miller C, Meehan K, et al. One-pass CO2 vs multiple-pass Er:YAG laser resurfacing in the treatment of rhytides: a comparison side-by-side study of pulsed CO2 and Er:YAG lasers. Dermatol Surg. 2001;27(8):709-715.

27. Liu A, Moy RL, Ozog DM. Current methods employed in the prevention and minimization of surgical scars. Dermatol Surg. 2011;37(12):1740-1746.

28. Ozog DM, Moy RL. A randomized split-scar study of intraoperative treatment of surgical wound edges to minimize scarring. Arch Dermatol. 2011;147(9):1108-1110.

29. Motamed S, Hasanpoor SE, Moosavizadeh SM, Arasteh E. Treatment of flexion contractures following burns in extremities. Burns. 2006;32(8):1017-1021.

30. Smith MA, Munster AM, Spence RJ. Burns of the hand and upper limb - a review. Burns. 1998;24(6):493-505.

31. Sklar LR, Burnett CT, Waibel JS, Moy RL, Ozog DM. Laser assisted drug delivery: a review of an evolving technology. Lasers Surg Med. 2014;46(4):249-262.
32. Waibel JS, Wulkan AJ, Shumaker PR. Treatment of hypertrophic scars using laser and laser assisted corticosteroid delivery. Lasers Surg Med. 2013;45(3):135-140.

33. Rkein A, Ozog D, Waibel JS. Treatment of atrophic scars with fractionated $\mathrm{CO} 2$ laser facilitating delivery of topically applied poly-L-lactic acid. Dermatol Surg. 2014;40(6):624-631.

34. Thangapazham RL, Darling TN, Meyerle J. Alteration of skin properties with autologous dermal fibroblasts. Int J Mol Sci. 2014;15(5):8407-8427.

35. Rodriguez-Menocal L, Salgado M, Davis S, et al. Percutaneous bone marrow transplantation using fractional ablative Erbium:YAG laser. PLoS One. 2014;9(3):e93004

36. Ranganathan K, Wong VC, Krebsbach PH, Wang SC, Cederna PS, Levi B. Fat grafting for thermal injury: current state and future directions. J Burn Care Res. 2013;34(2):219-226

37. Letada PR, Landers JT, Uebelhoer NS, Shumaker PR. Treatment of focal axillary hyperhidrosis using a long-pulsed Nd:YAG $1064 \mathrm{~nm}$ laser at hair reduction settings. J Drugs Dermatol. 2012;11(1):59-63.

38. Gratrix M, Hivnor C. Botulinum toxin A treatment for hyperhidrosis in patients with prosthetic limbs. Arch Dermatol. 2010;146(11):1314-1315.

39. Fearmonti R, Bond J, Erdmann D, Levinson H. A review of scar scales and measuring devices. Eplasty. 2010;10:e43. 